Infodiversidad

Sociedad de Investigaciones Bibliotecológicas

smf@rcc.com.ar

ISSN (Versión impresa): 1514-514X

ARGENTINA

\author{
2007 \\ Minerva del Ángel / Adolfo Rodríguez \\ LA PROMOCIÓN DE LA LECTURA EN MÉXICO \\ Infodiversidad, número 011 \\ Sociedad de Investigaciones Bibliotecológicas \\ Buenos Aires, Argentina \\ pp. 11-40
}

Red de Revistas Científicas de América Latina y el Caribe, España y Portugal 


\title{
La promoción de la lectura en México
}

\author{
MINERVA DEL ÁNGEL* \\ Dr. ADOLFO RODRÍGUEZ**
}

\section{Resumen}

Se analizan los esfuerzos realizados para promocionar y fomentar la lectura en México mediante la alfabetización de la población, la disponibilidad de materiales bibliográficos y el empleo de la tecnología de la información. Para ello se consideran las contribuciones del Sistema Educativo Nacional, las bibliotecas escolares y de aula y las bibliotecas públicas.

\section{Palabra clave}

lectura - México - Sistema Educativo Nacional - Bib. escolares - tecnología de la información - bibliotecas públicas

\begin{abstract}
The efforts carried out to promote and to foment the reading in Mexico are analyzed by means of the population's literacy, the availability of bibliographical materials and the employment of the information technology. Therefore, the contributions of the National Educational System, the school and classroom libraries and the public libraries are considered.
\end{abstract}

\section{Key words}

reading - Mexico - National Educational System - public libraries - school libraries - information technology

\section{Introducción}

La promoción de la lectura comprende el conjunto de actividades y acciones sistemáticas y continuas encaminadas a motivar, despertar o fortalecer el gusto e interés por la lectura y su utilización activa; es una práctica sociocultural no limitada al ámbito bibliotecario y

* Técnico Académico del Centro Universitario de Investigaciones Bibliotecológicas, CUIB UNAM, México. E-mail: minerva@cuibunam.mx Torre II de Humanidades, piso 11. Ciudad Universitaria 04510, México D.F.

${ }^{* *}$ Dr. Adolfo Rodríguez, Investigador del CUIB. Coordinador del Posgrado en Bibliotecología y Estudios de la Información, UNAM. E-mail: jadolfo@servidor. unam.mx 
escolar, que contribuye a la transformación del individuo y de la comunidad, facilitando el desarrollo social. ${ }^{1}$ La promoción se basa en el uso de los canales de comunicación para informar sobre la existencia de algo, en convencer a los posibles interesados de que usen o "adquieran" aquello que se promueve; por tanto incluye todas las actividades que divulgan a la organización, su oferta de productos o servicios, presentando al mercado o comunidad la información necesaria sobre su existencia y los beneficios que pueden esperarse sobre esos productos o servicios. ${ }^{2}$

En México se han propuesto diversas iniciativas para promover la lectura, desde el proyecto encabezado por José Vasconcelos a principios del siglo pasado, hasta el más reciente programa denominado Hacia un país de lectores. Todos los esfuerzos han girado fundamentalmente en dos sentidos, el primero es el desarrollo de grandes niveles de alfabetización y el segundo en poner libros al alcance de todos. Sin embargo, poco se ha hecho para formar ciudadanos con la habilidad, la capacidad y el compromiso de hacer que esos libros formen parte de su vida. A pesar de los logros alcanzados por el Sistema Educativo Nacional, éste no ha sido capaz de transmitir a la población la necesidad y el gusto por la lectura como una actividad de grandes beneficios individuales y sociales.

Estadísticamente hablando, en los últimos 70 años el Sistema Educativo Nacional ha logrado reducir el analfabetismo del 54 al 8.35 por ciento de 1930 a 2005 , y el rezago educativo del 94.8 al 43.9 por ciento de $1960^{3}$ a $2005 ;{ }^{4}$ condiciones que sólo se aplican a la población mayor de 15 años y que son más frecuentes entre las mujeres. Son analfabetas quienes no saben leer ni escribir, y están en rezago educativo quienes no tienen estudios secundarios y no asisten a la escuela.

${ }^{1}$ Edilma Naranjo Vélez, El bibliotecólogo como promotor de la lectura y sus bases pedagógicas (Tesis de Maestría en Bibliotecología, Universidad Nacional Autónoma de México, 2003), 109.

${ }^{2}$ Sueli Angelica do Amaral, Marketing da informação na Internet: ações de promoçao (Campo Grande, Brasil: UNIDERP, 2004), 73.

${ }^{3}$ Dirección General de Estadística, VIII Censo general de población 1960: resumen general (México, D.F.: Dirección General de Estadística, 1962), 1.

${ }^{4}$ INEGI, II Conteo de población y vivienda, 2005. Disponible en www.inegi.gob. $\mathrm{mx} / \mathrm{est} / \mathrm{default} . \mathrm{asp} ? \mathrm{c}=6224$ [Junio 27, 2006]. 
Por otra parte, la existencia del llamado analfabetismo funcional constituye un aspecto relevante que, aunque no ha sido medido a plenitud, debe ser seriamente considerado. Hasta el momento los esfuerzos realizados por cuantificar de manera directa este fenómeno no han sido suficientes, aunque al respecto IBBY México señala que más de la mitad de la población es analfabeta funcional, es decir que es incapaz de utilizar la lectura y la escritura como herramientas en su vida cotidiana:

Sin embargo, aun cuando la mayor parte de los mexicanos han aprendido a leer y escribir, muy pocos son capaces de comprender y manejar el lenguaje escrito, y de utilizarlo como herramienta de trabajo para la organización social, el aprendizaje o la diversión. El porcentaje de analfabetas funcionales alcanza un $60 \%$ de la población. Según estudios de la Unesco, nuestro país ocupa el lugar 107 en el mundo por su nivel y cantidad de lectura. ${ }^{5}$

En este nuevo siglo, los programas oficiales relacionados con la lectura se iniciaron con la publicación, en junio del año 2000, de la Ley de Fomento para la Lectura y el Libro expedida con el objeto de fomentar y promover la lectura. En este documento se estableció la creación de un Consejo Nacional de Fomento de la Lectura y del Libro responsable de realizar el Programa Nacional de Fomento a la Lectura que debería, entre otras cosas, elaborar "Paquetes didácticos de estímulo y formación de lectores, adecuados para cada nivel de la educación básica, dirigidos a educandos, docentes y padres de familia" y "Cursos de capacitación vinculados al trabajo editorial, gráfico, librero y bibliotecario". 6

El Consejo Nacional de Fomento de la Lectura y del Libro tendría entre sus atribuciones contribuir en la elaboración, seguimiento, evaluación y actualización del Programa, así como el desarrollo de sistemas integrales de información sobre la lectura, los derechos de autor y las bibliotecas disponibles para la consulta en red desde cualquier parte

${ }^{5}$ Asociación Mexicana para el Fomento del Libro Infantil y Juvenil, A. C. (IBBY México) http://www.ibbymexico.org.mx/ [Julio 3, 2006].

${ }^{6}$ Ley de Fomento para La lectura y el Libro [en línea], disponible en http://www. cddhcu.gob.mx/leyinfo/pdf/39.pdf [Noviembre 8, 2005]. 
del país; sistemas de información que no han podido construirse aun o al menos no con el nivel de descripción suficiente para reflejar con certidumbre la situación nacional.

Por su parte el Programa Nacional de Cultura 2001-2006 se propuso abatir de manera decisiva y constante los bajos índices de lectura mediante el concurso de toda la sociedad, desarrollando una política nacional del libro y la lectura que tomaría en consideración las necesidades y problemas específicos. Para ello se reconoció la urgencia de enmarcar la formación de lectores y la promoción de la lectura en la educación y la cultura. En este contexto la Secretaría de Educación Pública (SEP) y el Consejo Nacional para la Cultura y las Artes (Conaculta) asumieron responsabilidades.

\section{EI Sistema Educativo Nacional y las bibliotecas escolares}

El Programa Nacional de Lectura ${ }^{8}$ ha estudiado los ambientes escolares en virtud de que aportan datos interesantes sobre la relación entre educación y desarrollo de la habilidad lectora. El análisis de tales ambientes se ha dado en los tres niveles que integran la educación obligatoria: preescolar, primaria y secundaria.

Estos estudios han podido determinar que en preescolar son pocos los niños que tienen la posibilidad de participar de forma cotidiana en actos lectores y de escritura dentro de los jardines y en sus núcleos familiares. En la primaria se busca incorporar el enfoque funcional y comunicativo, que permita al niño aprender permanentemente y con independencia, así como actuar con eficacia e iniciativa en las cuestiones prácticas de la vida cotidiana; en este nivel se establece como propósito que los niños adquieran el hábito de la lectura y se formen como lectores que reflexionan sobre el significado de lo que leen y puedan valorarlo y criticarlo, que disfruten de la lectura y formen sus propios criterios de preferencia y de gusto estético. En la secundaria se

${ }^{7}$ Programa Nacional de Cultura 2001-2006, disponible en http://www.conaculta. gob.mx/programa/155.html [Abril 20, 2006].

${ }^{8}$ Programa Nacional de Lectura [en línea], disponible http://lectura.dgme.sep. gob.mx/documentos/pnl/html/pnl.html [Noviembre 8, 2005]. 
han empezado a crear necesidades generalizadas del uso de la lectura y la escritura en todas las áreas de estudio.

El discurso político sugiere que el Sistema Educativo Nacional da importancia fundamental a la lectura en el desarrollo de sus actividades y que en el marco que le brinda el Programa Nacional de Lectura busca promover la formación de lectores y escritores autónomos al introducir material bibliográfico en cada salón de clase de la educación básica; sin embargo, es un hecho que ha sido incapaz de integrarla de modo eficiente, nulificando con ello los esfuerzos por crear bibliotecas o colecciones en los planteles. La dotación de colecciones de calidad que respondan a las necesidades e intereses de la comunidad plantelaria fue considerada como un aspecto fundamental, aunque también se ha reconocido que su presencia no garantiza que sean usados e incorporados en la cultura escolar; por ello se concluye que la dotación de acervos debe concebirse sólo como una parte de la estrategia global de promoción de la lectura.

En el sitio web de la Secretaría de Educación Pública se señala entre los principales logros de 2001 a 2006 la conformación de 850 mil bibliotecas de aula con 464 títulos y 150 mil bibliotecas escolares con 225 títulos. $^{9}$

Sin embargo, de acuerdo con la información estadística publicada por el Instituto Nacional de Estadística, Geografía e Informática (INEGI), en 2001 existían 5,018 bibliotecas escolares y se estimaba que para el año 2005 el número había llegado a 5,301;10 siguiendo con el INEGI, la cantidad de escuelas de educación primaria y secundaria en 2005 se calculaba en $128,994 .{ }^{11}$ Resulta más que evidente la existencia de discrepancias entre la información de ambas instituciones. Otro aspecto interesante de analizar es el índice de infraestructura de los indicadores del Sistema Educativo Naciona ${ }^{12}$ el cual nos deja ver que en las es-

${ }^{9}$ SEP, Programa Nacional de Lectura, disponible en http://www.sep.gob.mx/ wb2/sep/Programa_Nacional_de_Lectura [Julio 5, 2006].

${ }^{10}$ INEGI, Bibliotecas según tipo, 1990-2005, disponible en http://www.inegi.gob. $\mathrm{mx} / \mathrm{est} / \mathrm{contenidos} / \mathrm{espanol} / \mathrm{rutinas} / \mathrm{ept}$.asp $\mathrm{t}=$ mcul03\&c $=3130$ [Julio 6, 2006].

${ }^{11}$ INEGI, Recursos humanos, materiales y financieros por nivel educativo, 1950 a 2005 disponible en: http://www.inegi.gob.mx/est/contenidos/espanol/rutinas/ept. asp? $\mathrm{t}=$ medu03\& $\mathrm{c}=3270$ [Julio 6, 2006].

${ }^{12}$ INEE, Panorama educativo de México: indicadores del Sistema Educativo Nacional, 2004. México : INEE, 2004, 241-244. Disponible en http://multimedia.ilce. 
cuelas primarias el 13.8 por ciento de las bibliotecas ${ }^{13}$ están en buenas condiciones; que en las escuelas primarias generales el porcentaje se eleva al 15.2 por ciento mientras que en las secundarias generales es del 17.5 por ciento y en las secundarias técnicas el 27 por ciento.

En realidad casi nada sabemos de las bibliotecas escolares mexicanas, no parece haber información pública respecto de sus colecciones, servicios, personal y programas. Se sabe que las escuelas primarias han sido dotadas con los "Libros del Rincón" y publicaciones del Consejo Nacional de Fomento Educativo, pero que por lo general no cuentan con espacios físicos ni con los recursos humanos capaces de poner en operación las bibliotecas escolares y de aula o salas de lectura. La educación secundaria por su parte no cuenta con el suficiente número de bibliotecas, pero esto es sólo parte del problema, hay que agregar que las bibliotecas poseen acervos limitados y no cuentan con personal capacitado a cargo de ellas, por lo que el concepto de biblioteca es, en el mejor de los casos, el lugar en donde se hacen tareas o se usan los libros para copiar alguna información. En la educación básica se tiene la idea de que una biblioteca es la colección y no los servicios que se deben ofrecer.

El predominio de esta situación indica que las bibliotecas escolares son vistas aún por el Sistema Educativo Nacional como meros apéndices de la escuela y no como parte crucial de la estrategia a largo plazo sobre la alfabetización y la educación. La biblioteca escolar debería ser considerada como un programa que requiere de una cuidadosa planeación y una constante inversión de recursos para mejorar los procesos de alfabetización y de educación, así como el gusto por la lectura entre estudiantes, maestros, administradores y padres de familia; un lugar en el que los estudiantes encuentren lecturas complementarias.

Respecto de las bibliotecas de aula se han enfatizado los datos estadísticos sin que se conozcan sus efectos a corto, mediano y largo plazos con relación a su objetivo establecido. Con este acercamiento no sólo se pretende valorar la infraestructura sino los procesos del sistema educativo, en el que debe realizarse el proceso de enseñar a aprender.

edu.mx/inee/indicadores_2004.htm [Julio 6, 2006].

${ }^{13}$ En las cifras a las que se hace referencia se consideraron las características ya sea en biblioteca escolar y/o de aula. 
Para IBBY México la introducción al proceso lector es de gran relevancia, la metodología didáctica tradicional -señala- enfatiza la adquisición de habilidades básicas, pero se ocupa poco del desarrollo de hábitos y actitudes positivas hacia la lectura. En otras palabras, se enseña a los niños la mecánica de la lectura pero no a querer leer. En el ciclo escolar 2005-2006 esta organización puso en marcha una nueva iniciativa para fomentar la lectura en la escuela pública, que consistió en trabajar directamente con los niños en los salones de clase y realizar una investigación sobre el impacto del acervo de las bibliotecas de aula.

Aunque IBBY México obtuvo el consentimiento de la SEP, el proyecto no es oficial. El objetivo de Nosotros entre libros -como se denominó al proyecto- es disminuir la desigualdad en la educación e incrementar los índices de lectura entre la población; antes de empezar el programa y al terminar el mismo se aplicaron evaluaciones para poder hacer comparaciones y medir el progreso.

La carencia de estudios que brinden elementos para evaluar el mayor o menor éxito de los esfuerzos realizados, así como las cifras $\mathrm{y}$ los indicadores mencionados sobre las bibliotecas escolares y sus condiciones, hablan de una situación difícil para la promoción de la lectura y la formación de lectores en los niveles básicos del Sistema Educativo Nacional.

\section{Niños y adolescentes: ¿analfabetas funcionales?}

El Instituto Nacional para la Evaluación de la Educación (INEE) aplicó una serie de pruebas nacionales para medir la comprensión de lectura en el ciclo escolar 2003-2004 en los grados terminales de primaria y secundaria $\left(6^{\circ}\right.$ y $3^{\circ}$ respectivamente).$^{14}$ Las escalas utilizadas tienen valores extremos de 200 y 800 puntos cada una, pero en los resultados no se encuentran valores inferiores a 300 ni superiores a 700 ni la indicación de nivel insuficiente, mínimo o alto.

${ }^{14}$ Instituto Nacional para la Evaluación de la Educación, Resultados de las pruebas nacionales de lectura y matemáticas por la modalidad educativa (México: INEE, 2005). 
No obstante, esta evaluación muestra los resultados por variante del sistema educativo, que resultan muy interesantes. Los resultados obtenidos en promedio por los alumnos de $6^{\circ}$ año de primaria son: escuelas privadas 568 puntos, escuelas urbanas públicas 497, escuelas rurales públicas 462 , cursos comunitarios 451 , educación indígena 425 , siendo el promedio nacional de 488 puntos. Los resultados obtenidos por los alumnos del $3^{\circ}$ de secundaria fueron: escuelas privadas 649 puntos, escuelas secundarias generales 565 , escuelas secundarias técnicas 564 , telesecundarias 524, y el promedio general fue de 564 puntos.

Durante los últimos años, la valoración de las habilidades lectoras ha abandonado el concepto reduccionista de la lectura; es decir ha transitado de la mera reproducción de letras y sílabas a un concepto mucho más profundo vinculado con la adquisición y uso del conocimiento:

Previamente, el énfasis en la política fue puesto en el efecto deletéreo del "analfabetismo": un acercamiento que probó ser inútil, puesto que falló en alertar al lector sobre el hecho de que todas las personas saben leer y escribir hasta cierto grado... La pregunta que la International Adult Literacy Survey (IALS) formuló a los individuos ya no fue “¿Puede usted leer?” sino “¿Qué tan bien lee usted?”.15

En México el INEE es el responsable del Programa Internacional para la Evaluación Estudiantil (PISA, por sus siglas en inglés) que entre otros aspectos mide el nivel de habilidad para la lectura entre jóvenes estudiantes de 15 años. La habilidad para leer es de suma importancia porque permite al individuo comprender y procesar todos los conocimientos a los que accede a lo largo de su vida. ${ }^{16}$

En PISA la aptitud lectora se mide en función de tres escalas: a) obtención de información, b) interpretación de textos, y c) reflexión y evaluación; los niveles de competencia tienen como objetivo clasificar el desempeño de los estudiantes y describir lo que son capaces de hacer. Los niveles de PISA son: ${ }^{17}$

${ }^{15}$ Literacy skills for the knowledge society: further results from the International Adult Literacy Survey (Ottawa : Human Resources Development Canada, c 1997), 11.

${ }^{16}$ Instituto Nacional para la Evaluación de la Educación, ¿Cómo están en lectura nuestros estudiantes de 15 años? [México : INEE, 2004?], 1.

${ }^{17}$ Ibid., 2-3. 


\begin{tabular}{|c|l|}
\hline Niveles & \multicolumn{1}{|c|}{ Características } \\
\hline $\begin{array}{c}\text { Nivel } 5 \text { (más } \\
\text { de } 625 \text { puntos) }\end{array}$ & $\begin{array}{l}\text { Los estudiantes son capaces de completar reactivos de } \\
\text { lectura sofisticados }\end{array}$ \\
\hline $\begin{array}{c}\text { Nivel } 4 \text { (de 553 } \\
\text { a 625 puntos) }\end{array}$ & $\begin{array}{l}\text { Los estudiantes son capaces de responder reactivos de } \\
\text { lectura difíciles }\end{array}$ \\
\hline $\begin{array}{c}\text { Nivel } 3 \text { (de 481 } \\
\text { a } 552 \text { puntos) }\end{array}$ & $\begin{array}{l}\text { Los estudiantes son capaces de manipular reactivos de } \\
\text { lectura de complejidad moderada }\end{array}$ \\
\hline $\begin{array}{c}\text { Nivel } 2 \text { (de } 408 \\
\text { a } 480 \text { puntos) }\end{array}$ & $\begin{array}{l}\text { Los estudiantes son capaces de responder reactivos básicos } \\
\text { de lectura }\end{array}$ \\
\hline Nivel 1 (de 335 & $\begin{array}{l}\text { Los estudiantes son capaces de responder correctamente } \\
\text { sólo los reactivos de lectura menos complejos como ubicar } \\
\text { un fragmento de información, identificar el tema principal } \\
\text { de un texto o establecer una conexión sencilla con el cono- } \\
\text { cimiento cotidiano. }\end{array}$ \\
\hline
\end{tabular}

Los estudiantes por abajo de los 335 puntos son incapaces de realizar el tipo de lectura básico que PISA busca medir, pero esto no significa que no tengan aptitud para la lectura, ya que los estudiantes pueden leer en el sentido técnico de la palabra. Sin embargo, enfrentarán dificultades para emplear la lectura como herramienta eficaz para ampliar y aumentar sus conocimientos en su transición al trabajo o para beneficiarse de una educación más amplia y de la oportunidad de aprender toda su vida.

En México existe una proporción considerable de jóvenes que no alcanzan las competencias básicas y se clasifican en el nivel 0 o por debajo del nivel 1 , y en contraste tan sólo un escaso número de estudiantes alcanzan el nivel 5. Ante tal situación el INEE ha reagrupado los niveles de competencia como medio de facilitar su análisis de la siguiente manera: competencia elevada (nivel 5), competencia intermedia (niveles 2, 3 y 4) e insuficiente (nivel 1 o menos), y el resultado es el siguiente:

Los puntajes promedio alcanzados por los estudiantes mexicanos muestran que sólo el 4.8 por ciento de los jóvenes se ubican en niveles 
de competencia alta, mientras el 52 por ciento se sitúa a niveles de competencia insuficiente. ${ }^{18}$

Los resultados de PISA también fueron desagregados por variante educativa, así el resultado promedio para las escuelas privadas es de 454 puntos y para las públicas 388 puntos.

\section{El analfabetismo, el rezago educativo y la promoción de la lectura}

Ya se ha mencionado que en los últimos 70 años el Sistema Educativo Nacional ha logrado reducir el analfabetismo -del 54 al 8.35 por ciento-y el rezago educativo - del 94.8 al 43.9 por ciento-y que ambas condiciones se aplican a la población mayor de 15 años.

En un trabajo sobre la educación de los jóvenes y adultos publicado por la Unesco, Galicia López señala que el sector en rezago educativo en México no demanda el acceso a opciones educativas formales ya que sus condiciones de sobrevivencia no les permiten visualizar la posibilidad de acceder a otros tipos de conocimiento y servicios educativos; estas personas tienen como ocupaciones personales el hogar y empleos de bajo nivel técnico y salarial en los sectores formales e informales. ${ }^{19}$

Es sabido que la población en rezago educativo es atendida por el Instituto Nacional para la Educación de los Adultos (INEA), el que, por ejemplo, ha tratado de coordinarse con los programas de desarrollo social para brindar atención a este sector de la población; estas gestiones no sólo no han sido exitosas sino que han puesto de manifiesto la falta de infraestructura y recursos financieros particularmente en el medio rural. Otras dependencias gubernamentales también ofrecen educación a personas jóvenes y adultas e incluso desarrollan programas de capacitación y productividad que se flexibilizan según el ritmo

${ }^{18}$ Ibid., 5.

${ }^{19}$ La educación de jóvenes y adultos en América Latina y el Caribe: hacia un estado del arte, disponible en http://www.unesco.cl/medios/biblioteca/documentos/educacion_jovenes_adultos_america_latina_caribe_hacia_estado_arte_2005. pdf?menu=/esp/atematica/alfayeduja/docdig/ [Julio 3, 2006]. 
de cada persona. Los esfuerzos realizados por esas instituciones han disminuido los índices de analfabetismo y rezago educativo, pero no han elevado el nivel ni la cantidad de la lectura en México, por ello se estima nuevamente necesaria una evaluación más objetiva de los programas, ya que hasta el momento las evaluaciones han enfatizado los datos estadísticos en vez de los efectos de los programas a corto, mediano y largo plazo.

Otros datos interesantes que proporcionaron las pruebas nacionales del INEE son aquellos sobre el contexto en el que se produce el aprendizaje, que ponen de manifiesto la relación del hogar con la educación y en particular con la lectura, y que se agrupan en dos conjuntos: el primero corresponde a los recursos económicos del hogar, o capital físico, y el segundo a la educación de los padres y otros elementos favorables al aprendizaje de los alumnos, o capital cultural:

Los datos muestran la relación entre contexto socioeconómico y logro educativo, conexión que también se observa en la información sobre el Índice Socioeconómico Internacional de Estatus Ocupacional (ISEI), que presenta cifras superiores en las escuelas privadas respecto de las públicas (51.99 contra 37.71). El índice relaciona la ocupación o trabajo que tienen los padres con sus ingresos, lo cual se refleja en un beneficio educativo de los hijos. ${ }^{20}$

Para valorar el capital físico se tomaron en cuenta tres indicadores: vivienda con luz eléctrica y refrigerador que indican mayor capital y piso de tierra que indica menor capital. Para el capital cultural se determinaron los siguientes indicadores: español como lengua que se habla en casa, alfabetización de la madre, existencia de enciclopedias y libros en el hogar. El que se hable una lengua indígena en el hogar no es indicio de menor capital cultural, pero dado que la lengua mayoritaria es el español, los alumnos de otra lengua materna se encuentran en desventaja. Los resultados se muestran en las tablas 1 y 2 .

${ }^{20}$ Ibid., 9. 


\section{TABLA 1. Indicadores de capital físico y cultural en las escuelas primarias}

\begin{tabular}{|c|c|c|c|c|c|c|}
\hline \multicolumn{7}{|l|}{ Capital Físico } \\
\hline & & & \multicolumn{4}{|c|}{ Escuelas Públicas } \\
\hline $\begin{array}{l}\text { Existencia en } \\
\text { el hogar de: }\end{array}$ & Total & Privadas & Urbanas & Rurales & $\begin{array}{l}\text { Indí- } \\
\text { genas }\end{array}$ & $\begin{array}{l}\text { Cursos } \\
\text { comunit. }\end{array}$ \\
\hline Luz eléctrica & 91.04 & 94.26 & 92.41 & 89.91 & 80.05 & 67.18 \\
\hline Refrigerador & 80.24 & 97.20 & 89.78 & 65.22 & 30.30 & 34.96 \\
\hline Piso de tierra & 12.20 & 1.01 & 5.21 & 22.48 & 51.85 & 46.91 \\
\hline
\end{tabular}

\begin{tabular}{|l|r|r|r|r|r|r|}
\hline \multicolumn{7}{|l|}{ Capital cultural } \\
\hline $\begin{array}{l}\text { Existencia en } \\
\text { el hogar de: }\end{array}$ & Total & Privadas & Urbanas & Rurales & $\begin{array}{c}\text { Indí- } \\
\text { genas }\end{array}$ & $\begin{array}{c}\text { Cursos } \\
\text { comunit. }\end{array}$ \\
\hline $\begin{array}{l}\text { Español como } \\
\text { lengua usual }\end{array}$ & 91.35 & 94.04 & 93.37 & 91.11 & 55.45 & 90.84 \\
\hline $\begin{array}{l}\text { Alfabetización } \\
\text { de la madre }\end{array}$ & 89.92 & 98.10 & 94.15 & 83.72 & 62.64 & 70.83 \\
\hline $\begin{array}{l}\text { Enciclopedias } \\
\text { libros }\end{array}$ & 76.97 & 95.60 & 82.87 & 63.40 & 63.63 & 56.40 \\
\hline
\end{tabular}

Adaptado de Instituto Nacional para la Evaluación de la Educación, Resultados de las pruebas nacionales de lectura y matemáticas por modalidad educativa (México : INEE, 2005), 8-9. 
TABLA 2. Indicadores de capital físico y cultural en las escuelas secundarias

\begin{tabular}{|l|r|r|r|r|r|}
\hline \multicolumn{1}{|l|}{ Capital Físico } \\
\hline & & & \multicolumn{3}{|c|}{ Escuelas Públicas } \\
\hline $\begin{array}{l}\text { Existencia en } \\
\text { el hogar de: }\end{array}$ & Total & Privadas & Generales & Técnicas & $\begin{array}{c}\text { Telese- } \\
\text { cundarias }\end{array}$ \\
\hline Luz eléctrica & 95.15 & 97.09 & 94.90 & 94.85 & 95.40 \\
\hline Refrigerador & 82.88 & 97.17 & 86.86 & 84.89 & 63.67 \\
\hline Piso de tierra & 8.10 & 0.72 & 5.55 & 9.02 & 16.25 \\
\hline
\end{tabular}

\begin{tabular}{|l|r|r|r|r|r|}
\hline \multicolumn{2}{|l|}{ Capital cultural } \\
\hline $\begin{array}{l}\text { Existencia en } \\
\text { el hogar de: }\end{array}$ & & & & & \\
\hline $\begin{array}{l}\text { Español como } \\
\text { lengua usual }\end{array}$ & 91.99 & 93.41 & 93.01 & 90.65 & 90.89 \\
\hline $\begin{array}{l}\text { Alfabetización } \\
\text { de la madre }\end{array}$ & 90.54 & 98.95 & 92.07 & 91.07 & 82.25 \\
\hline $\begin{array}{l}\text { Enciclopedias } \\
\text { y libros }\end{array}$ & 78.06 & 97.20 & 81.98 & 80.02 & 56.99 \\
\hline
\end{tabular}

Adaptado de Instituto Nacional para la Evaluación de la Educación, Resultados de las pruebas nacionales de lectura y matemáticas por modalidad educativa (México : INEE, 2005), 10.

Es evidente que el capital físico y cultural se ve determinado por el nivel educativo y el ingreso económico de los padres de familia, y que ambos constituyen el contexto social en el que se llevan a cabo los procesos de aprendizaje de los jóvenes estudiantes mexicanos; sin embargo existen muchos otros factores que deben tomarse en consideración.

De la información recolectada por PISA se desprende una relación entre entorno familiar y desempeño escolar. Por ejemplo, la situación laboral de los padres y el capital cultural de los hogares [...] tienen impacto en la lectura, así como el nivel educativo de los padres y el 
tipo de comunicación entre éstos y sus hijos. Asimismo, estudiantes que provienen de grupos étnicos minoritarios o son hijos de migrantes, pueden estar en desventaja y requerir atención especial para enfrentar un idioma y cultura que no son propios. ${ }^{21}$

\section{Conaculta: las bibliotecas públicas y las salas de lectura}

El Conaculta lanzó su programa Hacia un país de lectores que comprende la formación y capacitación de lectores de todas las edades, la producción, distribución y difusión de los libros, y la promoción intensiva de la lectura y el libro, en estrecha colaboración con el Sistema Educativo Nacional y los sectores social y privado. El programa se organiza articulando los programas y recursos del Consejo, de entre los cuales se destacan la Dirección General de Publicaciones y la Dirección General de Bibliotecas Públicas.

Además de un extenso programa de publicaciones, la Dirección General de Publicaciones ha establecido salas de lectura como una medida más para fomentar el gusto por leer, principalmente entre los jóvenes y los adultos. Las salas de lectura operan con colecciones variadas de libros en las que las personas, coordinadas por un promotor capacitado, leen, comentan y disfrutan la lectura a través de diversas actividades y del préstamo de libros.

De acuerdo con el resumen ejecutivo del Programa de Promoción de Lectura, de 1995 a 2005 se habían abierto 5,342 22 salas de lectura en la república mexicana y según sus indicadores de desempeño el promedio mensual de personas mayores de cinco años atendidas por cada sala fue de 80.82. Al relacionar los datos anteriores se observa que en promedio las salas atienden a 431,740.44 personas al mes, pero si esta cifra se relaciona con el total de la población de cinco y más años -que en 2005 fue de 90,266,425 $5^{23}$ mexicanos- observaremos que las salas de lectura están atendiendo tan sólo al 0.47 por ciento de la población. De la población atendida el 69.09 por ciento estudia, el 39.91 tiene la

${ }^{21}$ Ibíd., 10.

${ }^{22}$ Conaculta, Dirección General de Publicaciones, Promoción de la lectura, disponible en http://www.conaculta.gob.mx/dgp/promocion/index.html [Julio 4, 2006].

${ }^{23}$ INEGI, II Conteo de población y vivienda, 2005, disponible en http://www.inegi.gob.mx/est/contenidos/espanol/proyectos/conteos/conteo2005/default.asp?c $=6224$ [Julio 4, 2006]. 
primaria y el 22.87 por ciento la secundaria; el 32.87 por ciento tiene de cinco a 10 años y el 31.46 por ciento de 11 a 20 años.

Por las cifras anteriores se observa que los beneficiarios de las salas de lectura son fundamentalmente niños y adolescentes en edad escolar pero no los adultos y adultos jóvenes. No se pretende decir que esté mal, sino que no llega al segmento de la población compuesto de adultos.

Para contribuir al fomento de la lectura la Red Nacional de Bibliotecas Públicas amplió su cobertura y promovió distintas actividades que inciden en los procesos educativos y el hábito y el gusto por la lectura particularmente entre la población infantil y juvenil. El 70 por ciento de la población que se benefició de las actividades de la Red de Bibliotecas Públicas y el 80 por ciento que acudió a las actividades de fomento a la lectura fueron niños y jóvenes $;{ }^{24}$ al igual que en las salas de lectura, los adultos no participan ampliamente en estos programas.

Los programas desarrollados por las bibliotecas públicas para promover la lectura entre la población son Mis vacaciones en la biblioteca $\mathrm{y}$ los Talleres de Lectura que se estructuran en varias sesiones en torno a un tema central entre los usuarios de la misma biblioteca. Esta situación puede interpretarse como una debilidad de los programas debido a que sus esfuerzos no han logrado llamar la atención de los usuarios potenciales, entre los que se encuentra la población adulta.

No obstante, las bibliotecas públicas han reconocido la necesidad de fortalecer su función como formadoras de lectores habituales y autónomos, ya no sólo entre los niños, jóvenes y estudiantes, sino entre otros sectores de la población. ${ }^{25}$ Pero no se trata sólo de que la biblioteca pública reconozca su responsabilidad en la promoción de la lectura, sino que asuma un papel activo respaldado en procesos de planeación, organización, implantación y evaluación a corto, mediano y largo plazo que reflejen su capacidad para vincularse eficientemente con las comunidades y con el Sistema Educativo Nacional.

${ }^{24}$ Conaculta, Memoria 1995-2000. Tomo II, IV instituciones que conforman el Consejo Nacional para la Cultura y las Artes, 04. Dirección General de Bibliotecas Públicas, disponible en http://www.conaculta.gob.mx/memorias/dgbp.htm [Julio 4, 2006].

${ }^{25}$ Conaculta, Atlas de infraestructura cultural, disponible en http://sic.conaculta. gob.mx/atlas.php?estado_id=0\&estado_Id= [Julio 4, 2006]. 


\section{Tecnología de la información y promoción de la lectura}

La tecnología de la información (TI) ha sido introducida en los diversos ámbitos sociales, y la promoción de la lectura no ha sido la excepción. Durante los últimos años han surgido programas de promoción de la lectura apoyados en la tecnología de la información particularmente en bibliotecas escolares y públicas alrededor del mundo. Partiendo de diferentes contextos y perspectivas, se puede decir que la aplicación de la TI a las labores de promoción, en su sentido más elemental, ha consistido en la generación de listas de libros recomendados mediante el OPAC y el diseño digital de folletos y otros materiales promocionales con el fin de hacer disponibles los recursos impresos con que cuentan las bibliotecas.

Las bibliotecas escolares con mayores recursos humanos y tecnológicos han diversificado sus acciones en su propio sitio web, entre las que se encuentran: ${ }^{26}$

- Bookmarks que incluyen títulos de libros, o el OPAC de la biblioteca

- Listas de los nuevos títulos adquiridos por la biblioteca, títulos con características especiales o sugerencias para leer durante el verano

- Conversión de los folletos promocionales en archivos PDF para que los estudiantes puedan consultarlos

- Creación de ligas a reseñas y títulos nuevos

- Establecimiento de ligas a sitios web que tengan listas de libros sugeridos

- Digitalización de materiales creados por los jóvenes

- Realización de una encuesta en línea en la que los estudiantes voten por su libro favorito

- Diseño de un formato de recomendación de un libro

- Provisión de materiales en formato electrónico o e-libros.

Todos estos esfuerzos no han sido realizados en la soledad de la biblioteca, sino más allá; la biblioteca se ha relacionado con los profesores, con quienes ha compartido información sobre la importancia de que se dé tiempo a los estudiantes para seleccionar y leer libros.

${ }^{26}$ Mary Alice Anderson, "Reading promotion in the age of technology," MultiMedia \& Internet@Schools [en línea]11,no.6 (nov/dic 2004 ) : 29-30. Disponible EBSCOHost, Academic Search Premier, 14915715. 
En el plano de la biblioteca pública, la TI ha sido empleada para: ${ }^{27}$

- La colocación de listas de libros sugeridos con "probaditas" que enganchen a los usuarios hasta concluir la lectura, evidentemente en este caso se trata de material electrónico

- Un factor de éxito en la promoción de la lectura es relacionar al lector con el libro correcto, para ello algunas bibliotecas realizan entrevistas de referencia en línea. En este sentido las bibliotecas han diseñado formatos de solicitud de préstamo en línea y reseñas de libros también en línea

- Se incluyen reseñas de libros elaboradas por estudiantes y usuarios, lo que les permite compartir los libros que han disfrutado y leer libros que de otra manera no hubieran leído, además de motivar habilidades escritas

- Proyectos Read-In, que son aquellos donde las comunidades seleccionan de uno a varios libros y motivan a todos sus miembros a leerlo, en un periodo definido de tiempo. En estos programas se busca que la comunidad discuta el libro en clase, en la cafetería, en la biblioteca e incluso durante la cena; por ello la biblioteca mediante el OPAC informa al lector sobre los ejemplares disponibles, entre otros servicios que puede proporcionar a los lectores

- Club del libro en línea, se ha diseñado para aquellos que no tienen disponibilidad de horario. El punto de partida es la lista de títulos y las fechas para cada lectura y se caracteriza por operar a través de chat o de listserv.

Algunos otros esfuerzos han dado lugar a "hojas de lectura" o al diseño de una página web, que aunque vinculada con la biblioteca, su principal función es la promoción de la lectura. Estas hojas pueden contener listas de recomendaciones, reseñas e incluso el archivo histórico de las discusiones y listas anteriores.

La TI puede aplicarse para fomentar la comunicación entre lectores-lectores y lectores-escritores. El ciberespacio puede ser el punto

${ }^{27}$ Julieta Dias Fisher y Ann Hill, "Reading in the cyber age: getting teens wired to read!" Library Media Connection [en línea] 22, no. 3 (nov 2003), disponible EBSCOHost, Academic Search Premier, 11186918. 
de encuentro donde las personas se reúnan a chatear sobre una gran diversidad de temas: los libros que leen, sus autores favoritos y sugerirse lecturas. En estos foros pueden también participar celebridades y autores como ocurre en algunos países. En el ámbito educativo estas actividades permiten alcanzar dos objetivos: la promoción del uso de Internet y de las telecomunicaciones así como de la lectura: ${ }^{28}$

Internet combina muchos de los mejores elementos de la educación: los estudiantes aprenden rápidamente que deben ser capaces de leer, de escribir bien y de solucionar problemas en grupo para poder contribuir en proyectos en línea, y que además todo eso es divertido. ${ }^{29}$

Al respecto es pertinente subrayar que no es posible la participación de una persona en el ciberespacio sin la lectura, y que proyectos de esta naturaleza integran la práctica de la lectura con la escritura, conformándose en magníficas posibilidades de promover la comunicación y las colecciones que integran la biblioteca. ${ }^{30}$

Este acercamiento a la lectura y su promoción implica valorar la infraestructura y equipamiento del sistema educativo en el que se realizan las actividades de enseñanza-aprendizaje. Así, la disponibilidad de espacios apropiados para la atención de una demanda geográficamente dispersa, el equipamiento educativo y el estado de conservación y funcionalidad de la infraestructura, mobiliario y equipo en los planteles es también un indicador que debe tomarse en cuenta.

Se ha dicho ocasionalmente que la tecnología es "demasiado costosa" dadas las insuficiencias del financiamiento en el trabajo de instrucción, especialmente en los países desarrollados. Lo contrario también puede ser verdad: el trabajo de alfabetización (y la educación elemental más ampliamente) no puede permitirse ignorar el enorme potencial de la tecnología de la red y la educación a distancia, ya que de otra manera

28 “The Read In! goes global” Reading today [en línea] 14, no. 3 (dic 96/ ene 97), disponible EBSCOHost, Academic Search Premier, 9701294073.

${ }^{29}$ Ibíd

${ }^{30}$ Rachel Wadham, "Kids, books and the Web" Library Mosaics [en línea] 15, no. 2 (Mar/Abril 2004) : 20. 
la brecha entre la riqueza informativa y la pobreza informativa, la diferencia entre ricos y pobres, crecerá. ${ }^{31}$

Si tratamos de vincular la promoción de la lectura con la TI, los indicadores de infraestructura tecnológica (datos de 2002) podrían darnos una idea de la magnitud del fenómeno.

De acuerdo con los indicadores del Sistema Educativo Nacional se observa lo siguiente: ${ }^{32}$

1. La proporción de alumnos que tienen acceso a una computadora en la escuela o en su casa con respecto al total de los alumnos entrevistados es de 2.6

2. El promedio de alumnos por computadora en aquellas escuelas donde existe al menos una computadora con fines educativos es de 4.8

3. El porcentaje de docentes que utilizan computadoras en clase es de 10.2

4. La proporción de escuelas que cuentan con acceso a Internet respecto al total de escuelas con computadora es de 34.2

5. El porcentaje de escuelas que cuentan con programas electrónicos educativos oficiales es de 57.5

6. El porcentaje de escuelas que cuentan con programas electrónicos educativos comerciales es 71.1.

A pesar de que el porcentaje de escuelas que cuentan con Internet es elevado, esto no necesariamente significa que los alumnos tengan acceso a ella; la computadora e Internet han sido bien recibidos por las escuelas como apoyo a los procesos administrativos, se plantea esta posibilidad debido a que el 16.8 por ciento de los directores utilizan la computadora y el 34.7 por ciento de los responsables de medios tam-

${ }^{31}$ Daniel A. Wagner y Christopher Hopey, "Literacy, electronic networking” en Literacy: an international handbook, Daniel A. Wagner, Richard L. Venezky y Brian V. Street, eds. (Boulder : Westview Press, 1999), 480.

${ }^{32}$ Panorama educativo de México, indicadores del Sistema Educativo Nacional 2004, recursos materiales en las escuelas [en línea], 236-237, disponible en http:// multimedia.ilce.edu.mx/inee/pdf/indicadores/6\%20Ind\%20RM_2004.dfp [Octubre 26, 2005]. 
bién lo hacen. En ambos casos los niveles de uso sobrepasan aquellos observados en los alumnos y profesores.

Ante este panorama no resta más que incluir los indicadores sobre la importancia que se atribuye a la computadora y los medios de comunicación en las escuelas: el 4.2 por ciento de los directores de las escuelas muestran disponibilidad hacia las nuevas tecnologías, el 4 por ciento de los alumnos muestran una actitud positiva hacia las tecnologías de cómputo en la escuela, y el índice de influencia de medios de comunicación en los alumnos es del 3.6. Estos últimos datos hablan de que en las escuelas la TI aún no ha sido asimilada como la herramienta que es.

Respecto de la importancia de integrar la lectura y la tecnología de manera eficiente al currículo, la International Reading Association ha manifestado lo siguiente: ${ }^{33}$

La Internet y otras formas de tecnología de la información y comunicación (TIC) tales como procesadores de palabra, editores web, software para presentaciones y correo electrónico están regularmente redefiniendo la naturaleza de la alfabetización. Para ser completamente alfabetizados hoy en día, los estudiantes deben convertirse en expertos en las nuevas competencias de la TIC. Por lo tanto, los educadores tienen la responsabilidad de integrar efectivamente esas tecnologías al currículo de alfabetización para preparar a los estudiantes para el futuro que se merecen.

La International Reading Association cree que se puede hacer mucho para ayudar a los estudiantes a desarrollar las nuevas competencias que requerirán en el futuro. Creemos que los estudiantes tienen derecho a:

- maestros expertos en el uso efectivo de la TIC para enseñar y aprender;

- un currículo que integre las nuevas competencias de TIC en programas de instrucción;

- una instrucción que desarrolle las habilidades esenciales para un eficiente uso de la información;

${ }^{33}$ International Reading Association, Integrating literacy and technology in the curriculum (Newark, Delaware : International Reading Association, 2002). 
- una práctica en formación que incluya la lectura en Internet y la escritura usando procesadores de palabra;

- oportunidad para aprender segura y responsablemente a usar información y tecnología de la comunicación; y

- acceso equitativo a la TIC.

La International Reading Association declaró, con relación a su nueva posición respecto de la alfabetización y de la tecnología, que:

Las definiciones tradicionales de lectura, escritura, así como la definición tradicional de la mejor práctica en la instrucción -derivada de una gran tradición del libro y otros medios impresos-son en la actualidad insuficientes.

Hoy la definición de alfabetizado se ha expandido de las nociones tradicionales de lectura y escritura hasta incluir la habilidad para aprender, comprender, e interactuar con tecnología de un modo significativo. $^{34}$

Pero la TI no ha sido la única que se ha relacionado con los procesos de enseñanza-aprendizaje:

Las implicaciones de la tecnología en la alfabetización son algo paradójicas. Por un lado, la radio y la televisión han provisto alternativas a la lectura como un mayor vehículo de entretenimiento y adquisición de información; el teléfono nos ha hecho menos dependientes de la escritura como medio de comunicación a la distancia. Así existe la sensación hoy en día de que las personas que no saben leer o escribir están menos solas, mejor entretenidas y mejor informadas que sus contrapartes en el siglo XIX. Además, como consecuencia de los efectos de la tecnología en el lugar de trabajo, los requerimientos de instrucción para muchos empleos son considerablemente mayores de lo que lo fueron hace algunas décadas. ${ }^{35}$

${ }^{34}$ Julie Coiro, "Reading comprehension on the Internet: expanding our understanding of reading comprehension to encompass new literacies", Reading Teacher (2003) : 458.

${ }^{35}$ Raymond S. Nickerson, "Adult literacy and technology”, Visible language 10, no 3 (Verano 1985) : 330-331. 
Desde 1996, el Sistema Educativo Nacional ha estado trabajando en el proyecto denominado Red Escolar que se ha propuesto llevar a las escuelas de educación básica y normal un modelo tecnológico de convergencia de medios basado en el uso de la televisión, de Internet y la informática educativa. El objetivo del proyecto es proveer a la escuela con información actualizada y relevante y con un sistema de comunicación eficiente que permita a estudiantes y maestros compartir ideas y experiencias. ${ }^{36}$

Red Escolar fue diseñado con un modelo de equipamiento que incluía cuatro computadoras, un servidor, una impresora, un equipo de recepción de Edusat, una colección de discos compactos y una línea telefónica para conectarse a Internet; en este diseño se tomó en consideración que el promedio por grupo es de 40 integrantes. Red Escolar pretende fomentar el uso del correo electrónico, los foros de discusión, el intercambio de información, el desarrollo de habilidades para la investigación, confrontación de fuentes, redacción y argumentación, entre otros.

No se puede decir que la idea de la Red Escolar sea mala, pero sí tendríamos que decir que los recursos que se le asignan son verdaderamente minúsculos, y más aún después de conocer los indicadores sobre las condiciones de la infraestructura tecnológica de las escuelas. En ese contexto parecería una utopía pedir a un administrador del aula de medios que logre que cada grupo de la escuela acceda a Red Escolar por lo menos una hora a la semana.

Red Escolar es un importante esfuerzo por acercar contenidos de calidad a la educación básica mediante el empleo de la tecnología; sin embargo los principales problemas a que se enfrenta son la falta de infraestructura para la conectividad, la necesidad de capacitar a los profesores para el uso didáctico eficiente de la tecnología ante sus grupos y el desarrollo de contenidos. ${ }^{37}$

Al hacer referencia a los contenidos se abre una nueva dimensión para la reflexión que se puede expresar de la siguiente manera:

${ }^{36}$ Programa Nacional de Lectura [en línea], disponible http://lectura.dgme.sep. gob.mx/documentos/pnl/html/pnl.html [Noviembre 8, 2005].

${ }^{37}$ Red Escolar [en línea], disponible en http://redescolar.ilce.edu.mx/redescolar/ quees/indexquees.htm [Noviembre 8, 2005]. 
Actualmente la Internet y la World Wide Web muestran la promesa y los peligros de la publicación electrónica. Para aquellos con acceso a Internet, mucha información está disponible sin cargo, incluyendo documentos académicos y reportes técnicos así como documentos oficiales. Incluso estudiantes de secundaria y de grado pueden en algunos casos conducir investigación en Internet. Sin embargo, si lo hacen los profesores pueden tener menos control sobre sus trabajos que cuando la asignación se hacía sobre la base de libros de texto tradicionales o sobre los recursos de una biblioteca... Debido a que todo el material de la red se envía en el mismo formato a la pantalla de la computadora, no hay una distinción obvia entre los materiales ampliamente aceptados y los materiales que la sociedad encuentra problemáticos o perversos. En los materiales impresos, el canal de distribución y la calidad de la producción a menudo indicaban la aprobación o el rechazo social. Es fácil distinguir, por ejemplo, entre libros impresos por editores grandes para la venta en librería y los materiales pobremente editados distribuidos en la esquina de la calle, pero esta distinción obvia no existe en Internet. ${ }^{38}$

El desarrollo de contenidos que respalden o refuercen los planes y programas de estudio requiere de recursos humanos expertos en las diferentes asignaturas, con conocimiento en los contenidos y proyectos educativos y uso de las nuevas tecnologías, entre otros.

La Red Escolar también pretende contribuir al fomento de la lectura, a partir del tercer año de primaria y hasta el tercero de secundaria, al incluir textos, biografías de autores y preguntas generadoras.

En la información disponible sobre los programas de promoción de la lectura en las bibliotecas públicas no se menciona la aplicación de TI, lo que sugiere que hasta el momento ésta no ha ocurrido ni en la operación y promoción de los proyectos ni en la capacitación del personal. De hecho esta situación no debe parecer extraña, ya que el Programa de Acceso a Servicios Digitales en las Bibliotecas Públicas surge a partir del año 2002 con la finalidad de proporcionar acceso público y gratuito a la TIC a 1,763 bibliotecas de las 6,109 que existen. ${ }^{39}$

${ }^{38}$ Jonathan Anderson, "Information technologies and literacy", en Literacy : an international handbook, Wagner, Venezky y Street, op.cit., 465.

${ }^{39}$ Dirección General de Bibliotecas Públicas, Programa de acceso a servicios digitales en bibliotecas públicas [en línea], disponible http://dgb.conaculta.gob.mx/ 
Este programa contempla entre los servicios digitales el fomento de la lectura recreativa, informativa y formativa aunque no especifica en que forma. No es claro si a través de contenidos electrónicos o mediante la promoción de los servicios y colecciones de la biblioteca, o incluso de ambos.

El equipamiento de las bibliotecas, dada la variedad de servicios digitales que se pretende proporcionar a la comunidad, parece en esta etapa de implementación muy limitado, y aunque el proyecto se ha puesto en operación, los resultados aún no son visibles. Por lo anterior, plantear la promoción de la lectura en las bibliotecas públicas mexicanas mediante el empleo de la TI, en este momento significa explorar un escenario probable a largo plazo que representará un gran potencial de desarrollo.

\section{Para concluir}

A lo largo de este trabajo se ha señalado que gran parte de los esfuerzos realizados en la promoción de la lectura en México se han enfocado en dos aspectos fundamentales: la alfabetización y la disponibilidad de materiales. Sin embargo, hasta el momento no se tienen datos que describan el éxito o efectividad de los mismos en términos de un incremento cualitativo y/o cuantitativo de la lectura en México. La reducción del analfabetismo y la disponibilidad de materiales no implica que la práctica de la lectura en México se amplíe, particularmente cuando el analfabetismo funcional crece. La ausencia de evaluación nos coloca en desventaja en la medida en que no podemos determinar si se ha logrado avance en esta tarea titánica.

Además del Sistema Educativo Nacional, las bibliotecas públicas

y escolares juegan un papel importante en la formación del hábito por la lectura, en el que se reconoce su responsabilidad en la promoción de la lectura como parte de su labor social. Por ello, los programas de promoción de la lectura deben formar parte de un plan articulado que necesariamente implica el análisis del entorno educativo y un trabajo

pasd.htm [Octubre 27, 2005]. 
conjunto para garantizar fuentes de financiamiento, estabilidad y continuidad de los proyectos a todos los niveles y en todos los sectores.

La evidencia sobre la aplicación exitosa de la TI a programas de promoción de la lectura en otros países no hace sino confirmar el gran potencial de la tecnología de la información en los procesos educativos y culturales de la sociedad moderna. Sin embargo, nuestra realidad nacional pone de manifiesto que México no cuenta aún con la infraestructura que le permita entrar plenamente a promover la lectura mediante el empleo de TI.

Entre las variables que inciden de forma directa sobre el éxito de los programas de promoción de la lectura se han señalado las siguientes:

1. La dificultad que experimenta el Sistema Educativo Nacional en la formación de lectores

2. La dificultad del Sistema Educativo Nacional para operar eficientemente bibliotecas escolares, bibliotecas de aula y salas de lectura. La dotación de materiales bibliográficos es estéril en la medida en que las bibliotecas no operan

3. La dificultad del Sistema Educativo Nacional en incorporar las bibliotecas de manera efectiva a las actividades de enseñanzaaprendizaje

4. El deficiente equipamiento en TI en las escuelas

5. El déficit financiero que sufre la educación en México, que causa entre otras cosas el deterioro de la infraestructura educativa, y en especial aquella relacionada con bibliotecas, computadoras e Internet

6. La falta de oportunidades para que los niños y jóvenes mexicanos tengan acceso equitativo a la TIC y

7. La falta de oportunidades para que los niños y jóvenes mexicanos aprendan a usar información y TI.

Las bibliotecas públicas mexicanas han implementado un programa permanente de fomento a la lectura, del cual se conocen estadísticas pero no los resultados de su evaluación; esto implica la carencia de un referente que le permita ajustar sus programas a las necesidades específicas de las diversas comunidades a las que sirve. La incorpora- 
ción de TI a la biblioteca pública, al ser tan reciente, se encuentra en estado larvario y posiblemente transcurrirán aún algunos años antes de consolidar su infraestructura y aplicarla de manera eficiente a sus procesos de promoción de la lectura. Así por ejemplo la información sobre bibliotecas públicas que cuentan con OPAC o un sitio en la red, no está disponible, por tanto no se conocen las condiciones sobre las cuales se podrían diseñar los programas de promoción de lectura basados en TI.

En general existe un gran déficit de información relacionada con los programas oficiales de fomento y promoción de la lectura, que no nos permite conocer con un cierto grado de certidumbre la situación nacional.

Por otra parte se reconoce la importancia de la participación de la familia en la educación de sus miembros; al respecto los niveles educativos ubican a poco menos de la mitad de la población mayor de 15 años con una formación menor a los nueve años de escolaridad.

El acceso a la TI en el hogar, la escuela y la biblioteca pública es marginal, lo cual debe ser contemplado al establecer programas de promoción ya que por su naturaleza la promoción se apoya en medios de comunicación de amplio espectro para llegar a su mercado real o potencial. La proporción de niños y jóvenes que usan Internet, con relación al total de la población mayor de seis años, es de 4.35 por ciento, un dato que valdría la pena mantener siempre en mente.

Sería fabuloso que México se convirtiera en un país de lectores por decreto, pero sabemos que eso jamás ocurrirá. Los programas nacionales están llenos de muy buenas intenciones y mucho esfuerzo; sin embargo no han probado con certeza ser efectivos y posiblemente esta situación se deba a que se expide una "receta" general para todos, olvidándonos de que la necesidad y el gusto por la lectura es un asunto individual y personal. De ahí que el estudio de las comunidades particulares sea crucial para tener éxito e inducir efectivamente un cambio de actitud. Si bien es cierto que la red de bibliotecas públicas no puede asumir las responsabilidades de la escuela de educación básica y de la biblioteca escolar, también lo es que dada su experiencia puede servir como modelo para la construcción de una red de bibliotecas escolares que asuma de manera efectiva su responsabilidad social. 


\section{Obras consultadas}

Amaral, Sueli Angelica do. Marketing da informação na Internet : ações de promoçao. Campo Grande, Brasil : UNIDERP, 2004.

Anderson, Jonathan. "Information technologies and literacy". En Literacy: an international handbook. Daniel A. Wagner, Richard L. Venezky y Brian V. Street, eds. Boulder: Westview Press, 1999.

Anderson, Mary Alice. "Reading promotion in the age of technology". MultiMedia \& Internet@Schools [en línea], 11, no. 6 (nov/dic 2004): 29-30. Disponible EBSCOHost, Academic Search Premier, 14915715.

Asociación Mexicana para el Fomento del Libro Infantil y Juvenil, A. C. (IBBY México) http://www.ibbymexico.org.mx/ [Julio 3, 2006].

Coiro, Julie. "Reading comprehension on the Internet: expanding our understanding of reading comprehension to encompass new literacies." Reading Teacher (2003) : 458.

Conaculta. Atlas de infraestructura cultural [en línea]. Disponible en http://sic.conaculta.gob.mx/atlas.php?estado_id=0\&estado_Id= [Julio 4, 2006].

man el Consejo Nacional para la Cultura y las Artes, 04. Dirección General de Bibliotecas Públicas [en línea]. Disponible en http:// www.conaculta.gob.mx/memorias/dgbp.htm [Julio 4, 2006].

Conaculta. Dirección General de Bibliotecas Públicas. Acciones para el fomento a la lectura en las bibliotecas públicas [en línea]. Disponible http://dgb.conaculta.gob.mx/fomento.htm [Noviembre 27, 2005].

Programa de acceso a servicios digitales en bibliotecas públicas [en línea]. Disponible http://dgb.conaculta.gob.mx/pasd. htm [Octubre 27, 2005].

Conaculta. Dirección General de Publicaciones. Promoción de la lectura [en línea]. Disponible en http://www.conaculta.gob.mx/dgp/ promocion/index.html [Julio 4, 2006].

Dirección General de Estadística. VIII Censo general de población 1960: resumen general. México, D. F. : Dirección General de Estadística, 1962. 
La educación de jóvenes y adultos en América Latina y el Caribe: hacia un estado del arte [en línea]. Disponible en http://www.unesco. $\mathrm{cl} /$ medios/biblioteca/documentos/educacion_jovenes_adultos_america_latina_caribe_hacia_estado_arte_2005.pdf?menu=/esp/atematica/alfayeduja/docdig/ [Julio 3, 2006].

Fisher, Julieta Dias y Ann Hill. "Reading in the cyber age: getting teens wired to read!" Library Media Connection [en línea] 22, no. 3 (nov 2003). Disponible EBSCOHost, Academic Search Premier, 11186918.

INEGI. Bibliotecas según tipo, 1990-2005 [en línea]. Disponible en http://www.inegi.gob.mx/est/contenidos/espanol/rutinas/ept. asp? $\mathrm{t}=$ mcul03\&c=3130 [Julio 6 julio 2006].

- Indicadores seleccionados sobre nivel de escolaridad, promedio de escolaridad, aptitud para leer y escribir y alfabetismo, 1960 a 2005 [en línea]. Disponible en http://www.inegi.gob.mx/est/ contenidos/espanol/rutinas/ept.asp? $\mathrm{t}=$ medu09\&c $=3277$ [Octubre 27, 2005].

. Recursos humanos, materiales y financieros por nivel educativo, 1950 a 2005 Disponible en: http://www.inegi.gob.mx/ est/contenidos/espanol/rutinas/ept.asp? $\mathrm{t}=$ medu03\&c $=3270$ [Julio 6, 2006].

. II Conteo de población y vivienda, 2005 [en línea]. Disponible en www.inegi.gob.mx/est/default.asp?c=6224 [Junio 27, 2006].

Instituto Nacional para la Evaluación de la Educación. ¿Cómo están en lectura nuestros estudiantes de 15 años?, [México : INEE, 2004?]. . Resultados de las pruebas nacionales de lectura y matemáticas por la modalidad educativa. México : INEE, 2005.

-_-_-_- Panorama educativo de México : indicadores del sistema educativo nacional, 2004. México: INEE, 2004. Disponible en http://multimedia.ilce.edu.mx/inee/indicadores_2004.htm [Julio 6, 2006].

International Reading Association. Integrating literacy and technology in the curriculum. Newark, Delaware: International Reading Association, 2002.

Kirsch, Irwin S. "Measuring adult literacy". En Toward defining literacy. Newark, Delaware: International Reading Association, 1990. 
Lankshear, Colin. "Illiteracy, improper literacy and the development of the underclass". En Towards successful schooling. Londres : Falmer Press, 1990.

Literacy skills for the knowledge society: further results from the International Adult Literacy Survey. Ottawa : Human Resources Development Canada, $c 1997$.

Ley de Fomento para la Lectura y el Libro [en línea]. Disponible en http://www.cddhcu.gob.mx/leyinfo/pdf/39.pdf [Noviembre 8, 2005].

Love, Lucy y Hannah Richens. "First steps into the library". Literacy Today (septiembre 2003) : 11.

Manifiesto de la IFLA/UNESCO sobre la biblioteca pública, 1994 [en línea]. Disponible en http://www.ifla.org/VII/s8/unesco/span.htm [Octubre 27, 2005].

Manifiesto UNESCO/IFLA sobre la biblioteca escolar [en línea]. Disponible en http://unesco.org/webworld/libraries/manifestos/school_manifesto_es.html [Octubre 27, 2005].

Naranjo Vélez, Edilma. El bibliotecólogo como promotor de la lectura y sus bases pedagógicas (Tesis de Maestría en Bibliotecología, Universidad Nacional Autónoma de México, 2003).

Nickerson, Raymond S. "Adult literacy and technology". Visible language 10, no. 3 (verano 1985): 330-331.

Panorama educativo de México, indicadores del Sistema Educativo Nacional 2004, recursos materiales en las escuelas [en línea]. Disponible en http://multimedia.ilce.edu.mx/inee/pdf/indicadores/ 6\%20Ind\%20RM_2004.dfp [Octubre 26, 2005].

Petit, Michèle. Nuevos acercamientos a los jóvenes y la lectura. Tr. de Rafael Segovia y Diana Luz Sánchez. México : Fondo de Cultura Económica, 1999.

Programa Nacional de Cultura 2001-2006 [en línea]. Disponible en http://www.conaculta.gob.mx/programa/155.html [Abril 20, 2006]. Programa Nacional de Lectura [en línea]. Disponible http://lectura. dgme.sep.gob.mx/documentos/pnl/html/pnl.html [Noviembre 8, 2005]. 
"The Read In! goes global" Reading today [en línea] 14, no. 3 (dic 96/ene 97). Disponible EBSCOHost, Academic Search Premier, 9701294073.

Red Escolar [en línea]. Disponible en http://redescolar.ilce.edu.mx/redescolar/quees/indexquees.htm [Noviembre 8, 2005].

Secretaría de Educación Pública. Programa Nacional de Lectura [en línea]. Disponible en http://www.sep.gob.mx/wb2/sep/Programa Nacional_de_Lectura [Julio 5, 2006].

Wadham, Rachel. "Kids, books and the Web." Library Mosaics [en línea] 15, no. 2 (marzo/abril 2004) : 20.

Wagner, Daniel A. y Christopher Hopey, "Literacy, electronic networking" en Literacy: an international handbook, Daniel A. Wagner, Richard L. Venezky y Brian V. Street, eds. Boulder : Westview Press, 1999. 